\title{
Niereninsuffizienz
}

\section{Ist der Cystatin-C-Test genauer als das Plasma-Kreatinin?}

Die Zahl niereninsuffizienter Patienten wird in den kommenden Jahren rasch anwachsen. Die diagnostischen Werkzeuge zur Früherkennung sind bislang unzureichend. Seit Januar ist der neue Parameter Cystatin C Leistung der gesetzlichen Kassen. MMW hat Professor Walter Hofmann, Chefarzt des Departments für Klinische Chemie am Städtischen Klinikum München, nach den Vorteilen des neuen Parameters gefragt.

MMW: Das Plasma-Kreatinin ist ein vergleichsweise wenig empfindlicher Parameter zur Erfassung der Nierenfunktion. Was zeichnet den neuen Parameter Cystatin C aus?

Hofmann: Mit der klassischen Bestimmung des Kreatinins im Plasma zur Erfassung der glomerulären Filtrationsrate (GFR) kann man milde Nierenfunktionsstörungen nicht erkennen. Das ist der Bereich zwischen 60 und $90 \mathrm{ml} / \mathrm{min} / 1,73 \mathrm{~m}^{2}$, der sogenannte kreatininblinde Bereich. Die Ermittlung der Kreatinin-Clearance liefert hier nur eine partielle Lösung.

Der Cystatin-C-Wert ist dagegen kaum abhängig von Einfluss- und Störgrößen und zeigt auch im kreatininblinden Bereich eine Nierenfunktionsstörung an. Damit wird eine diagnostische Lücke geschlossen.

\section{MMW: Was ist Cystatin C? \\ Hofmann: Cystatin C ist ein kleinmo- lekulares Protein, ein Inhibitor von Cy- stein-Proteinasen. Zwischen dem 1. und 6o. Lebensjahr ist die Konzentration sehr konstant. Cystatin C wird frei glomerulär filtriert und proximal tubulär nahezu voll- ständig reabsorbiert. Nimmt also die Zahl der funktionsfähigen Nephrone ab, „staut“ sich das Cystatin C zurück. Nach dem 60. Lebensjahr steigt Cystatin C kontinuierlich an, denn die Zahl funktionsfähiger Ne- phrone kann mit dem Alter um 30-40\% abnehmen. Der Parameter spiegelt damit - im Unterschied zum Kreatinin - den phy- siologischen Alterungsprozess wider.}

MMW: Gibt es Störgrößen, die die Aussagekraft des Cystatin-C-Werts beeinträchtigen?
Hofmann: Schilddrüsenfunktionsstörungen und eine hoch dosierte Glukokortikoidtherapie können den Cystatin-C-Wert beeinflussen. Zu den Störgrößen Adipositas und Rauchen ist die Datenlage noch uneinheitlich.

MMW: Nun gibt es ja die vereinfachte MDRD*-Formel nach Levey zur Abschätzung der GFR, mit dem Einflussgrößen auf das Kreatinin berücksichtigt werden. Warum bleibt man nicht einfach dabei? Hofmann: Die Basis dieser Formel ist nunmal der Kreatininwert mit den entsprechenden Störfaktoren, auch wenn Alter, Geschlecht und die Rasse einbezogen werden. Validiert ist die MDRD-Formel zudem nur bei niereninsuffizienten Patienten mit einer GFR zwischen 30 und $60 \mathrm{ml} / \mathrm{min} /$ $1,73 \mathrm{~m}^{2}$. Die Formel passt jedoch nicht für Normalpersonen, für Menschen über 80

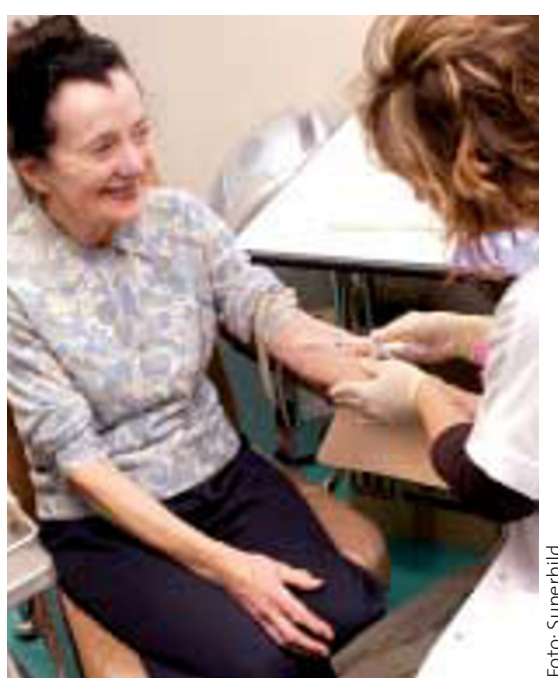

Bei älteren Patienten und Kindern ist der Cystatin-C-Test erste Wahl.

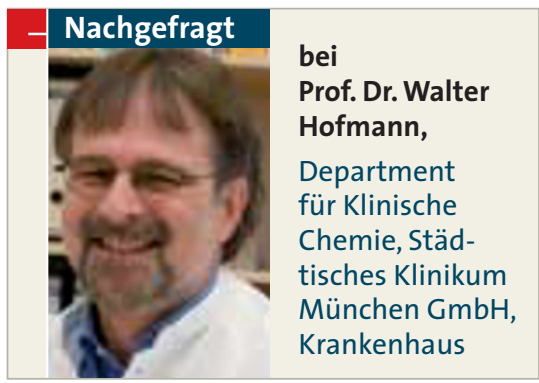

Jahre oder für Kinder. Und sie ist für viele Krankheitsgruppen nicht validiert. Cystatin C ist dem Plasma-Kreatinin überlegen, wenn es auch noch nicht die ideale Messgröße zur Abschätzung der GFR ist.

MMW: Warum braucht man auch bei Cystatin C eine Rechenformel zur Abschätzung der GFR?

Hofmann: Seit Jahrzehnten gehen Ärzte mit der GFR um und haben ein Gefühl dafür entwickelt. Die Rechenformel ermöglicht eine individuell noch genauere Aussage als der nackte Cystatin-C-Wert.

MMW: Nun ist der Cystatin-C-Test vergleichsweise teuer. Aus praktischer Sicht stellt sich die Frage: Wann nutze ich welchen Parameter?

Hofmann: Um sensitiv eine Nierenfunktionsstörung im Sinne eines möglichen frühen Funktionsverlustes detektieren zu können, ist für mich der Cystatin-C-Test die erste Wahl, besonders aber bei speziellen Patientenkollektiven wie Kindern sowie bei älteren Patienten mit deutlich reduzierter Muskelmasse.

Neben den Plasmamessgrößen zur Erfassung einer Nierenerkrankung darf man die Urindiagnostik nicht außer Acht lassen, wenn man Nierenerkrankungen ausschließen oder differenzieren möchte.

${ }^{*}$ MDRD - Modification of Diet in Renal Disease Study Group

(GFR $\left(\mathrm{mL} / \mathrm{min} / 1,73 \mathrm{~m}^{2}\right)=186^{*}(\mathrm{CREA})-1,154^{*}$ (Alter) $-0,203^{*}(0,742$ bei Frauen)

- Interview: Dr. Thomas Meißner

Literaturtipps:

W. Hofmann: MDRD-Formel und Cystatin C. Verbesserungen zur Erfassung der glomerulären Filtrationsrate? J Lab Med 2005;29(3):139-145

B. Holdt, P. Schuff-Werner: Labordiagnostische Beurteilung der Nierenfunktion im Alter. J Lab Med 2008; 32(2):43-56 